\title{
The gut microbiome modulates gut-brain axis glycerophospholipid metabolism in a region-specific manner in a nonhuman primate model of depression
}

\author{
Peng Zheng ${ }^{1,2,3,4} \cdot$ Jing $\mathrm{Wu}^{5} \cdot$ Hanping Zhang ${ }^{1,2,3} \cdot$ Seth W. Perry ${ }^{4} \cdot$ Bangmin Yin $^{1,2,3} \cdot$ Xunmin Tan $^{1,2,3} \cdot$ \\ Tingjia Chai ${ }^{1,2,3} \cdot$ Weiwei Liang $^{6} \cdot$ Yu Huang ${ }^{1,2,3} \cdot{\text { Yifan } \mathrm{Li}^{1,2,3} \cdot \text { Jiajia Duan }^{5} \cdot \text { Ma-Li Wong }^{4}{ }^{4} \text {. Julio Licinio }}^{4}$. \\ Peng Xie ${ }^{1,2,3}$
}

Received: 27 December 2019 / Revised: 9 April 2020 / Accepted: 20 April 2020 / Published online: 6 May 2020

(c) The Author(s), under exclusive licence to Springer Nature Limited 2020

\begin{abstract}
Emerging research demonstrates that microbiota-gut-brain (MGB) axis changes are associated with depression onset, but the mechanisms underlying this observation remain largely unknown. The gut microbiome of nonhuman primates is highly similar to that of humans, and some subordinate monkeys naturally display depressive-like behaviors, making them an ideal model for studying these phenomena. Here, we characterized microbial composition and function, and gut-brain metabolic signatures, in female cynomolgus macaque (Macaca fascicularis) displaying naturally occurring depressive-like behaviors. We found that both microbial and metabolic signatures of depressive-like macaques were significantly different from those of controls. The depressive-like monkeys had characteristic disturbances of the phylum Firmicutes. In addition, the depressive-like macaques were characterized by changes in three microbial and four metabolic weighted gene correlation network analysis (WGCNA) clusters of the MGB axis, which were consistently enriched in fatty acyl, sphingolipid, and glycerophospholipid metabolism. These microbial and metabolic modules were significantly correlated with various depressive-like behaviors, thus reinforcing MGB axis perturbations as potential mediators of depression onset. These differential brain metabolites were mainly mapped into the hippocampal glycerophospholipid metabolism in a regionspecific manner. Together, these findings provide new microbial and metabolic frameworks for understanding the MGB axis' role in depression, and suggesting that the gut microbiome may participate in the onset of depressive-like behaviors by modulating peripheral and central glycerophospholipid metabolism.
\end{abstract}

These authors contributed equally: Peng Zheng, Jing Wu

These authors jointly supervised this work: Julio Licinio, Peng Xie

Supplementary information The online version of this article (https:// doi.org/10.1038/s41380-020-0744-2) contains supplementary material, which is available to authorized users.

Julio Licinio

licinio@earthlink.net

$\triangle$ Peng Xie

xiepeng@cqmu.edu.cn

1 Department of Neurology, The First Affiliated Hospital of Chongqing Medical University, Chongqing, China

2 NHC Key Laboratory of Diagnosis and Treatment on Brain Functional Diseases, Chongqing Medical University, Chongqing, China

\section{Introduction}

Major depressive disorder (MDD) affects millions of individuals globally [1], with significant economic and social costs. Current understanding of the underlying MDD pathology focuses on various hypotheses of molecular brain dysfunctions [2]. However, antidepressant therapies based on these prevailing theories only alleviate symptoms in

3 Chongqing Key Laboratory of Neurobiology, Chongqing, China

4 Department of Psychiatry, College of Medicine, SUNY Upstate Medical University, Syracuse, NY, USA

5 The M.O.E. Key Laboratory of Laboratory Medical Diagnostics, the College of Laboratory Medicine, Chongqing Medical University, Chongqing, China

6 Department of Neurology, Yongchuan Hospital University, Chongqing, China 
about $40-50 \%$ of MDD patients [3, 4], suggesting that the existing hypotheses are not sufficient to reflect the heterogeneous pathophysiological mechanisms of MDD. Thus, it is of great relevance to explore novel potential molecular mechanisms of depression.

To date, rodents have served as the main model for investigating the molecular basis of depression, and have yielded many milestone research findings. However, rodent models of MDD frequently precipitate depressive behaviors by subjecting the animals to various physical stressors $[5,6]$, whereas in humans, MDD usually occurs under social and psychological stress [7-9], and these pathogenic features are difficult to reproduce in traditional rodent models. Thus, identifying animal models in which depression develops under relatively natural conditions, that better mimic the human experience, might be expected to yield important new insights into MDD pathology. Previously, we have developed a system for evaluating, defining, and classifying depressive-like (DL) and other behaviors of captive cynomolgus monkeys (Macaca fascicularis) $[10,11]$. Consistent with Shively et al.'s findings [12], we identified a hierarchically subordinate subgroup of monkeys that display DL behaviors. The majority of subordinates display DL behaviors that appear to arise from the continual stress and harassment these animals suffer from more dominant animals in the social hierarchy [13, 14]. As a chronic mild-to-moderate stressor, the constant social stress experienced by these primates has characteristics comparable with the kinds of underlying stressors that may precipitate MDD. Moreover, the biologic changes observed in these animals are similar to those found in MDD patients $[15,16]$. Thus, the DL monkey paradigm may be a useful new model for studying the pathogenesis of MDD.

The gastrointestinal tract contains thousands of resident microorganisms that together make up the gut microbiome, which has been increasingly shown to regulate many aspects of health and disease. Recent evidence suggests that gut microbiota modulate brain function and behaviors via the "microbiota-gut-brain" (MGB) axis [17, 18]. Our groups recently reported that MDD was associated with an altered gut microbiome [19], and this change was relatively specific relative to the microbial composition of bipolar disorder and schizophrenia [20, 21]. Using fecal transplantation experiments, we found that germ-free mice transplanted with "MDD microbiota" displayed depressive behaviors. Extending these studies, herein we investigate how the gut microbiome may contribute to depression onset via the MGB axis. The DL macaque model is especially suitable for these experiments because the monkey gut microbiome is more similar to that of human than the rodent gut microbiome, and less affected by confounding factors such as the living environment, diet, and comorbidities [22].
Since MDD is characterized by multiple disturbances of the gut microbiome, high-throughput technologies such as metagenomics and metabolomics are especially well-suited for capturing the molecular basis of depression. Metagenomic methods can interrogate the structure and function of the gut microbiome [23], and metabolomics provides supplemental functional readout of microbial activity [24]. Combining metagenomics with metabolomics is a wellestablished strategy to understand how the gut microbiome shapes the host. The weighted gene correlation network analysis (WGCNA) helps identify the molecular pathways that are co-regulated in a functionally coherent fashion [25]. These clusters or modules represent a single unit that can be associated with clinical or behavioral phenotypes. Using this method, Sousa et al. [26], successfully identified mRNA modules with similar variation across regions and species in both human and macaque brains.

Here, we first compared the microbial composition and function of DL and healthy control (HC) macaques to evaluate whether the DL monkey model was associated with gut microbiome disturbances. Comparative metabolomics were used to capture the functions of the altered gut microbiome by systematic analysis of relevant biological samples, including peripheral (fecal, intestinal wall, serum, and liver) and central (hippocampus, prefrontal cortex, and amygdala) specimens, from the DL and HC groups. Secondly, WGCNA was applied to identify key molecular modules and explore the correlation of microbial and metabolic modules with DL behaviors. Finally, these altered metabolites in the three brain regions were mapped to canonical metabolic pathways to identify the key pathways modulated by gut microbiome at the functional levels.

\section{Materials and methods}

\section{Ethics statement}

This study was performed in accordance with the recommendations in the "Guide for the Care and Use of Laboratory Animals" of the Institute of Neuroscience at Chongqing Medical University (\#20100031). All work involving nonhuman primates was conducted in accordance with NIH guide for the care and use of laboratory animals and with the recommendations of the Weatherall report, "The use of nonhuman primates in research" [27]. We also followed nc3r recommendations (https://www.nc3rs.org.uk/) by using the minimum number of depressed monkeys and agematched controls: based on the data of previous nonhuman primate studies $[28,29]$, and our work on behavioral and biochemical measures in $M$. fascicularis, the sample sizes ( $n=6 /$ every group) were chosen [11]. The M. fascicularis facilities, housing and primate laboratories used in this study 
are accredited by the Association for Assessment and Accreditation of Laboratory Animal Care. Monkeys were housed in an environmentally controlled facility $\left(22 \pm 1^{\circ} \mathrm{C}\right.$ temperature, $50 \pm 5 \%$ relative humidity and $12 \mathrm{~h}$ light $/ 12 \mathrm{~h}$ dark cycle with lights on at 7:00).

\section{Behavioral observation and tissue preparation}

The protocol for selecting animals displaying naturally occurring DL behaviors was as previously described [11]. Briefly, we surveyed the populations from 20 enclosures, and identified typical DL and corresponding HC monkeys. We observed them in the home enclosure for each macaque, termed the "free enclosures test" [10] (see Supplementary Methods). We also established a "social interaction test" to evaluate social communication behaviors (see Supplementary Methods), which was performed in separate cages. All the 12 identified macaques were adult female, and were ruled out for disease by veterinary examination. The procedures of macaque tissue preparation were presented in Supplementary Methods.

\section{S rRNA gene sequence analysis}

16S rRNA gene amplicon library preparation and sequencing were performed as described previously [19, 30] (see Supplementary Methods). Raw fastq files were quality-filtered by Trimmomatic and merged by FLASH [31]. In brief, raw fastq files were de-multiplexed, trimmed to remove lowquality bases, and paired-end $3^{\prime}$ overlapping mates were merged. Chimeric sequences were identified by UCHIME (https://www.drive5.com/usearch/manual/uchime_algo.html), then removed from subsequent analyses. Operational taxonomic units (OTU) were picked at $97 \%$ similarity using UPARSE (version $7.1 \mathrm{http}$ ://drive5.com/uparse/). The taxonomy of each $16 \mathrm{~S}$ rRNA gene sequence was analyzed by RDP Classifier algorithm (http://rdp.cme.msu.edu/).

Alpha diversity was calculated to estimate the microbial communities' diversity, including species richness indices (Ace and Chao) and species diversity indices (Shannon) [19]. Beta diversity analysis was performed to visually evaluate the difference of bacterial communities between DLs and HCs using Partial least-squares discriminant analysis (PLS-DA) [32]. The key bacterial taxa responsible for discrimination between two groups were identified using linear discriminant analysis Effective Size (LEfSe) [33].

\section{Comparisons of metabolite profiles from the $M$. fascicularis}

Details of the metabonomic methods were similar to our previously published protocols [19, 21]. Briefly, peripheral and brain tissue samples were prepared by homogenization, dissociation, and centrifugation, and serum samples were collected and centrifuged twice [19]. The gas chromatography-mass spectrometry and liquid chromatography-mass spectrometry data were used to characterize the metabolic changes in DLs relative to HCs. Discrimination of metabolites between DL and HC samples was analyzed and visualized using PLS-DA [24]. The differential metabolites responsible for discrimination between the two groups were identified on SIMCA, with the significance threshold of variable importance plot (VIP) $>1.0$ and $P$ values $<0.05$.

\section{Metagenomic analysis of fecal samples}

The metagenomic libraries were prepared and sequenced according to our previous protocols in Shanghai Majorbio Bio-pharm Technology Co., Ltd [19, 21] (see Supplementary Methods). Full details of the pipeline used to process and analyze metagenomic data are available. Threshold exclusion in sequence quality control and genome assembly was performed as we described previously [19, 21]. Herein, reads that were identified belonging to the $M$. fascicularis' reference genome were removed using BMA (Bayesing model averaging). The unit of gene abundance was unified using reads per kilobase million.

The function of nonredundant genes was annotated with the Kyoto Encyclopedia of Genes and Genomes (KEGG) (http://www.genome.jp/kegg/). We aligned the metagenomic genes onto KEGG GENES using BLASTP (V2.2.28, http://blast.ncbi.nlm.nih.gov/Blast.cgi), and annotated the genes with KEGG Orthology (KO) Based Annotation System, V2.0. Expectation value was set as 1e -5 in BLAST. We analyzed Metagenomic species (MGS) to de novo identify various microbial species without the complete reference genomes for each species. The canopy clustering method was used as described by Nielsen et al. [34], and performed with in-house scripts. The candidate genes for assembly were the different genes ( $n=230,868$, $P<0.05$, Wilcoxon signed-rank test) between DLs and HCs. All the candidate genes generated "seed" genes randomly, and then the "seed" genes were involved in coabundant gene (CAG) groups in which genes had similar abundance profiles (Pearson correlation coefficient $>0.9$ ). Each CAG group contained at least three genes, and $90 \%$ of the canopy genes came from more than three samples, each MGS contained at least 700 genes. The phylogenetic origin of MGS was identified by blasting genes in clusters against the NCBI (National Center for Biotechnology Information) nonredundant catalog and requiring that at least $50 \%$ of the genes had a best hit to the same phylogenetic group. 


\section{Weighted gene co-expression network analysis (WGCNA)}

WGCNA was used to identify key phenotype-related metagenomic and metabolic modules based on correlation patterns [25] (see Supplementary Methods). Associations between DL behavioral phenotypes and modules were calculated with Pearson correlation coefficients. The modules that significantly $(P \leq 0.01)$ associated with at least one DL phenotype were identified as potential phenotype-driven modules. Genes in each metagenomic module were annotated on KEGG; KOs were enriched in KEGG reference pathway, then the maps were classified into major functions. Metabolites in each module were annotated on Human Metabolome Database (http://www.hmdb.ca/) and Lipid Maps (http://www.lipidmaps.org/) for avoiding missing important metabolites, then the annotated metabolites were classified into major categories.

\section{Statistical analysis}

Statistical analyses on behaviors were carried out using SPSS version 18 (SPSS, Chicago, IL, US) and R studio (version 3.5.2, 2018) unless otherwise described. LEfSe analysis was used to identify the different OTUs by estimating the effect of the abundance of each OTU (LDA $>2$ and $P$ value $<0.05$ ). SIMCA-P software 11.0 was used to perform the PLS-DA analysis. Based on the literature, two statistical standards were used to identify differential metabolites between the two groups. Only metabolites with VIP $>1.0$ and significant difference by $t$-test $(P<0.05)$ were identified as key variations responsible for discrimination between the DL and HC groups. The investigators were not blinded to the group classification while analyzing the data.

\section{Results}

\section{Behavioral characteristics of the DL monkey model}

Based on our previously established protocol for DL macaque selection [10,11], we identified six typical DL and six HC monkeys among more than 300 macaques in 20 enclosures. The behaviors of the enrolled monkeys were videotaped (2366 min of free-enclosure tests and $720 \mathrm{~min}$ of social interaction tests) and quantified. We quantified the duration of each behavior (huddle, ingestion, sit alone, locomotion, amicable, and communication, Fig. 1a, b and Supplementary Table 1).

We found that except for ingestion, the remaining behaviors were significantly different between the DL and HC groups (Fig. 1c-h). Specifically, compared with HC,
DL monkeys were characterized by increased duration of huddling and sitting alone behaviors, and decreased duration of amicable, locomotion, and communication activities (Fig. 1c-g). These findings were similar to our previous results [10], confirming the effectiveness of this strategy in screening DL macaques in captivity.

\section{Gut microbial composition of HC and DL monkeys}

Using 16S ribosomal RNA (rRNA) gene sequencing, we first compared the gut microbial communities of DL and HC monkeys. In total, we obtained 635,259 high-quality reads across all samples with an average length of 437.94 base pairs (bp). These reads were clustered into 955 OTUs at $97 \%$ sequence similarity. A Venn diagram showed that 842 of 955 OTUs were common to the two groups, while 51 and 62 OTUs were unique to the HC and DL groups, respectively (Supplementary Fig. S1a). Within-sample $(\alpha)$ phylogenetic diversity analysis showed that there was no difference between two groups (Supplementary Fig. S1b).

\section{Gut microbiome alterations in DL monkeys}

A $\beta$-diversity analysis was performed to determine whether gut microbial compositions of DL monkeys were significantly different from those of controls. We found a robust difference in gut microbial composition between the two groups (Fig. 2a). The relative abundance of gut microbes at the family level was shown in Fig. 2b. Using LEfSe analysis, we identified 28 differential OTUs responsible for this discrimination (Fig. 2c and Supplementary Table 2). Compared with HCs, the DLs were characterized by 14 increased OTUs, mainly belonging to Veillonellaceae (5 OTUs) and Lachnospiraceae (5 OTUs), and 14 decreased OTUs mainly belonging to Ruminococcaceae (5 OTUs), at the family level. Interestingly, the majority of the OTUs $(25 / 28,89.28 \%)$ belonged to the phylum Firmicutes.

In addition, we found that 26 OTUs mainly belonging to the Ruminococcaceae, Lachnospiraceae, and the Veillonellaceae families were significantly linked with DL behaviors, especially huddling, sitting alone, and amicable and locomotion activities (Fig. 2c). Interestingly, 24 of 26 $(92.31 \%)$ OTUs that correlated with behavioral phenotypes belonged to Firmicutes. These findings highlight that disturbances of the phylum Firmicutes are a hallmark of the DL monkey model.

\section{MGS comparison between DL and HC monkeys}

Next, gut microbiome compositions of the DL monkeys were analyzed using whole-genome shotgun sequencing of cecum stool samples. The 12 fecal samples across both 
a

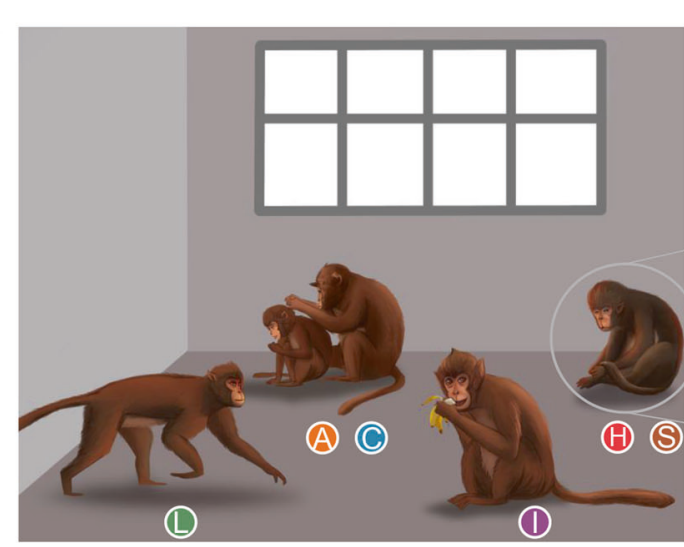

Representative A Amicable C Communication $($ Huddle behaviors b

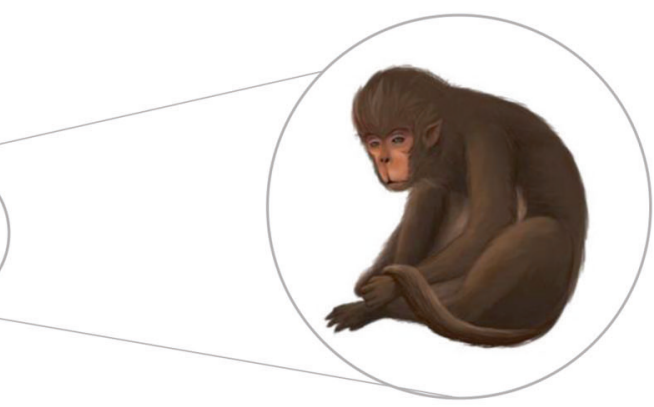

Depression-like M. fascicularis
C

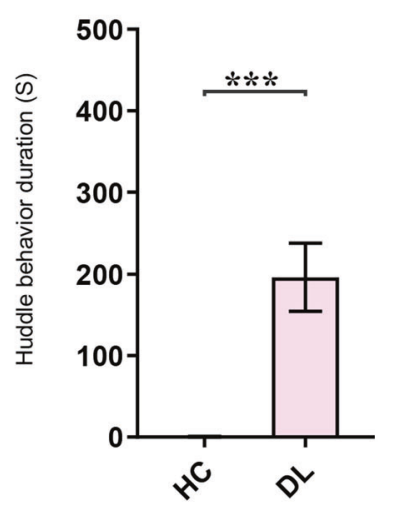

f

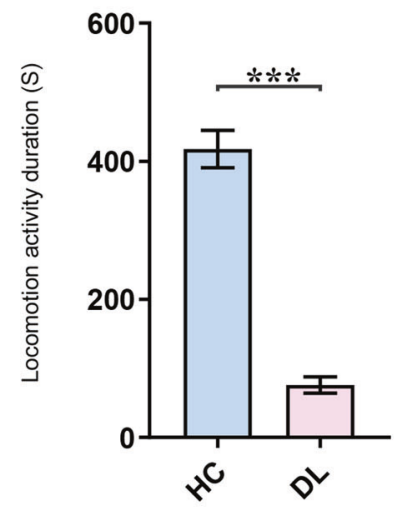

d

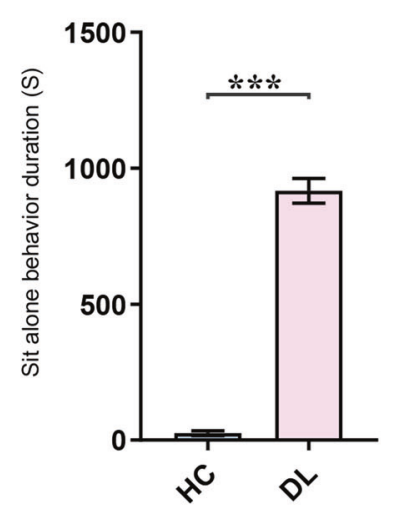

g

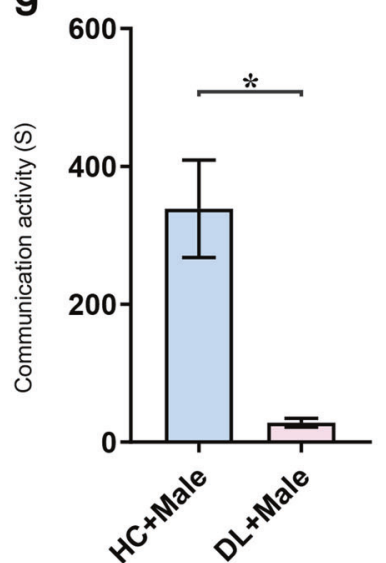

e

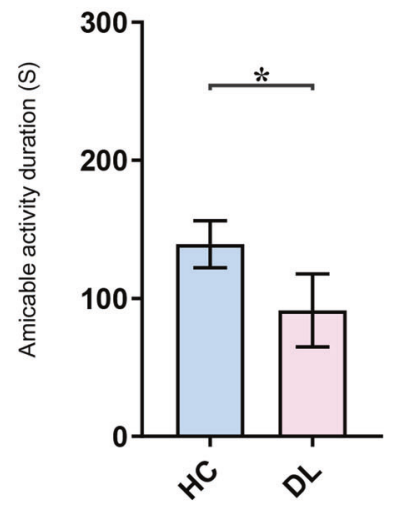

h

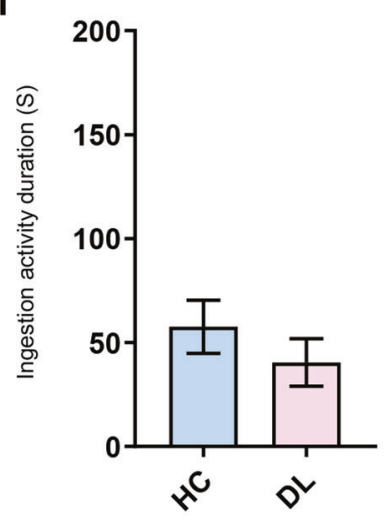

Fig. 1 Behavioral phenotypes of healthy (HC) versus depressivelike (DL) M. fascicularis. a Representative behaviors of DL and HC M. fascicularis under natural conditions. b Unique naturally-occurring DL behavior of $M$. fascicularis. c-f Compared with healthy controls, depression-like $M$. fascicularis displayed increased duration of huddle and sit-alone behaviors, and decreased duration of amicable and locomotion activities (DL, $n=6$; HC, $n=6$; $* P<0.05$, ** $P<0.01$, $* * * P<0.001$, two-sided Student's $t$ test; bars show mean $\pm \mathrm{SEM}$ ).
Videotape data were collected in 3 days and analyzed by NOLDUS software (every data point was defined as duration of behaviors over 3 days). $g$ In the sociability test, the DL group spent less time on communication activities with a male than the HC group (DL, $n=6$; HC, $n=6$; $* P<0.05, * * P<0.01, * * * P<0.001$, two-sided Student's $t$ test; bars show mean \pm SEM). $\mathbf{h}$ There was no difference of duration of ingestion activity. Videotape data were analyzed by NOLDUS software. 


\section{a}

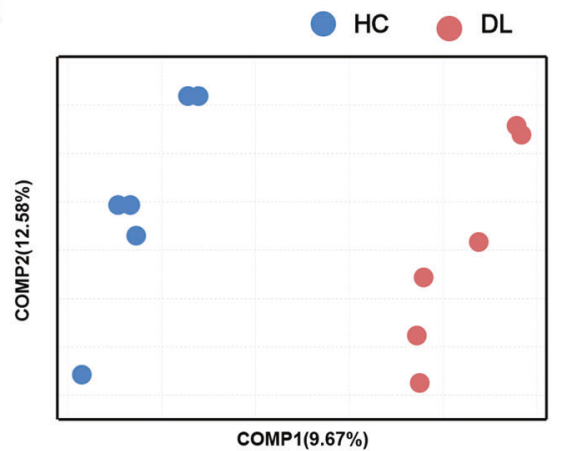

b

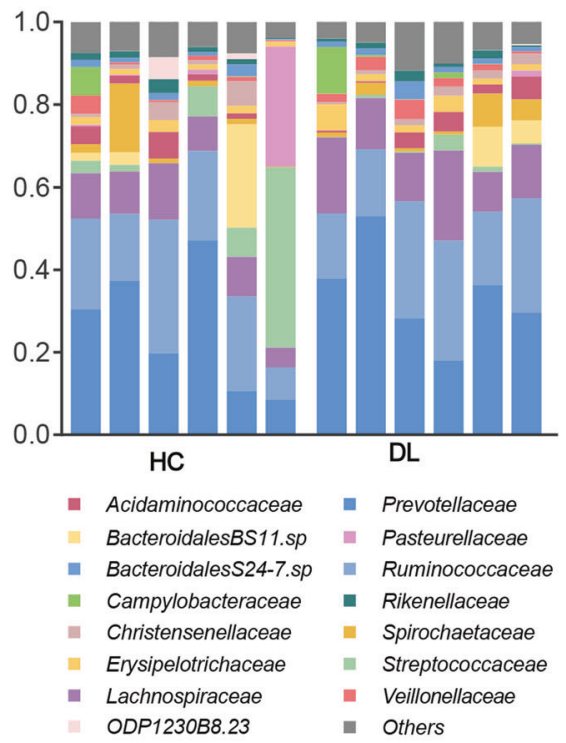

C

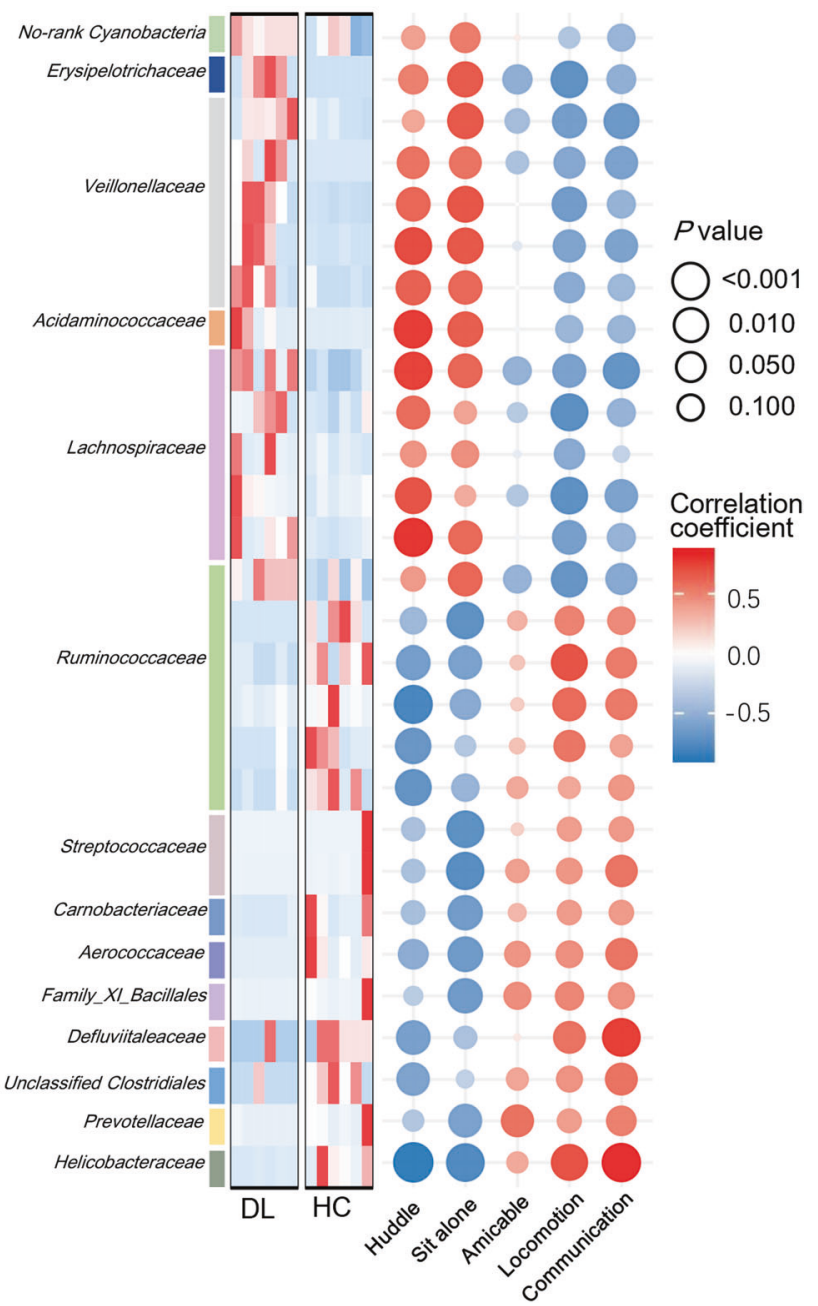

analysis (LDA > 2.0). All OTUs (raw) were sorted by taxa, and were classified at family level. The intensity of color in the middle heatmap (blue to red) indicates the normalized abundance score for each OTU. The size and color of each point in scatter plot shows the $P$ values of Spearman's correlation (ranging from $1.5 \mathrm{e}-4$ to 1 ) and values of correlation coefficient (ranging from -0.882 to 0.838 ) between differential OTUs and DL behaviors, respectively. groups yielded an average of $15,814,587,756$ bp per sample after quality control. From the filtered sequence data, we identified 37,912,983 predicted genes. Then we merged the predicted genes to 26,286 clusters and formed a nonredundant gene set for the subsequent analysis.

According to the correlation of abundance (Pearson correlation coefficient $r>0.9$ ), nonredundant genes in all samples were clustered into CAG groups. The CAG groups containing over 700 genes were identified as MGS. Consequently, 230,868 different genes were included in the analysis, defining 5657 CAG groups and 1330 MGS. The 1330 MGS contained each 700-137,714 genes, with a gene median of 1744 (Fig. 3a); these MGS were then mainly annotated to phylum (46.9\%) and order level (40.7\%)
(Fig. 3b). In total, there were 50 MGS with significant differences between DLs and HCs (Fig. 3c), which were primarily concentrated in the phylum Firmicutes (89.6\%). Furthermore, we analyzed the correlation between MGS and characteristic DL behaviors, and found that 48 MGS significantly correlated with DL behaviors, mainly belong to the phylum Firmicutes (85.42\%, 41/48; Fig. 3d).

\section{Perturbed microbial modules in DLs}

WGCNA analysis was used to identify the microbial modules. This analysis clustered 18 modules from 230,868 different genes, and the number of microbial genes in each module ranged from 963 to 32198 

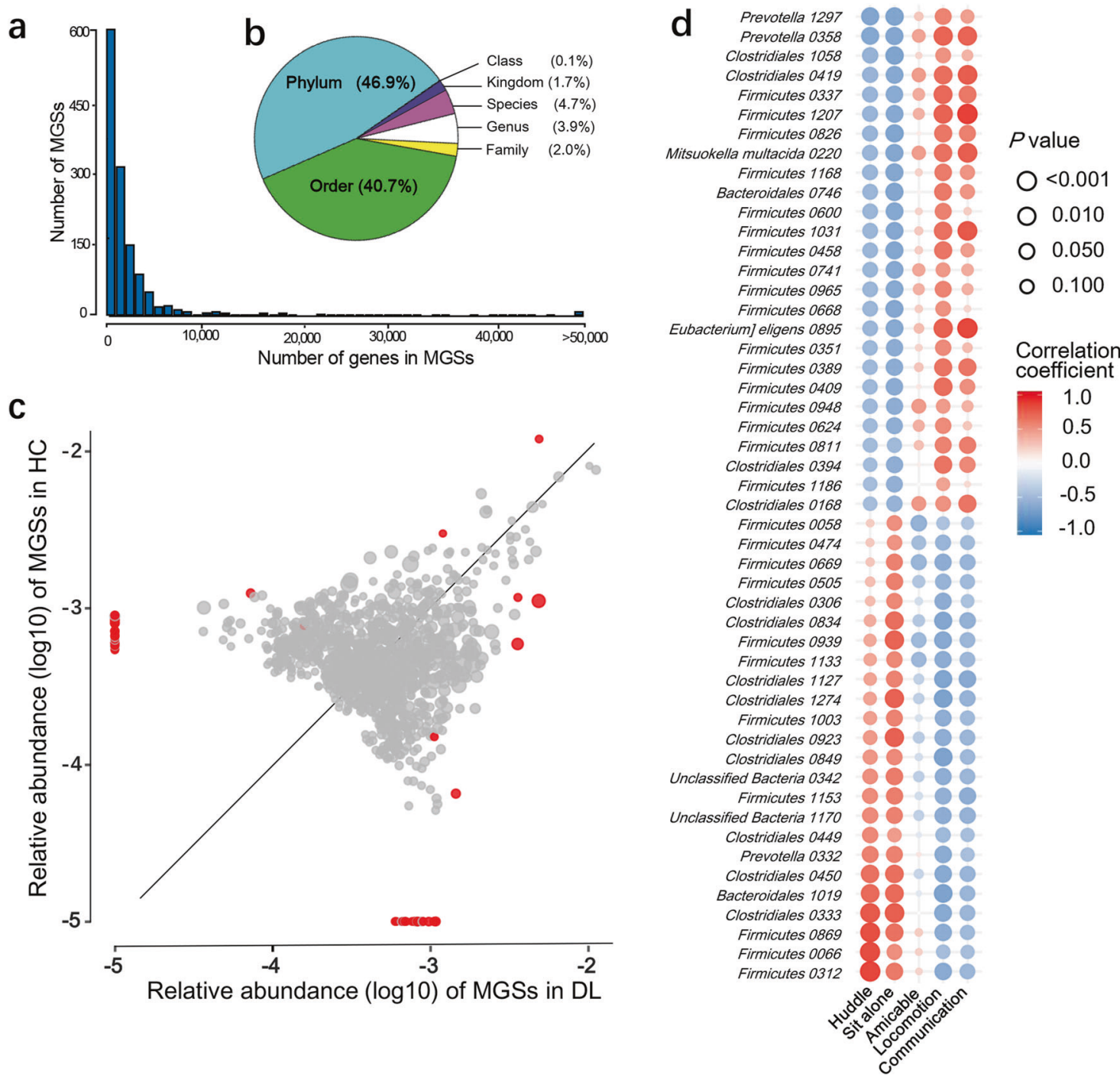

Fig. 3 Metagenomic species (MGS) differences in healthy (HC) versus depression-like (DL) $M$. fascicularis. a Histogram of the number of genes in MGS $(n=1330$, gene counts 700-103,023). b Pie chart of the taxonomic annotation level of MGS. c Scatter plot of median MGSs abundance in DL $(n=6)$ and HC $(n=6)$ macaque. Gray points represent MGSs which were not significantly changed between groups; red points represent the differentially expressed

(Supplementary Table 3a). Subsequent Pearson correlation analysis identified three significant associations between metagenomic modules and DL behaviors: the green-yellow module (M) (correlation coefficients $r=$ $0.7, P=0.01)$ was associated with huddling, and the cyan $\mathrm{M}(r=0.76, P=0.004)$ and the light-cyan $\mathrm{M}(r=-0.78$, $P=0.003)$ were associated with amicable activities (Fig. 4a and Supplementary Table 3b-d). Functional analysis revealed that the microbial genes in these modules mainly belonged to energy, neurotransmitter, nucleotide, and lipid metabolism pathways (Supplementary Fig. 2a-c). The lipid-related pathways identified from metagenomic WGCNA modules clustered on fatty acyls,
MGSs in the DL relative to the $\mathrm{HC}$ group $(P<0.05$, Wilcoxon rank sum test). d Association between 50 differential MGSs and DL behaviors. The size and color of each point in scatter plot shows the $P$ values of Spearman's correlation (ranging from $8.9 \mathrm{e}-5$ to 0.98 ) and correlation values of coefficient (ranging from -0.701 to 0.893 ) between differential MGSs and DL behaviors, respectively.

glycerophospholipids, sphingolipid, glycerolipids, and glycosphingolipid metabolism (Fig. 4b).

\section{Perturbed metabolic modules of the MGB axis in DL monkeys}

Next, non-targeted metabolomics analysis was performed to detect which metabolic pathways within the clustered microbial modules related to the MGB axis. We found that the metabolic signatures of peripheral (fecal, intestinal wall, serum, and liver) and central (hippocampus, prefrontal cortex, and amygdala) samples in DL were significantly different from those of HC monkeys (Supplementary Fig. 3). In 


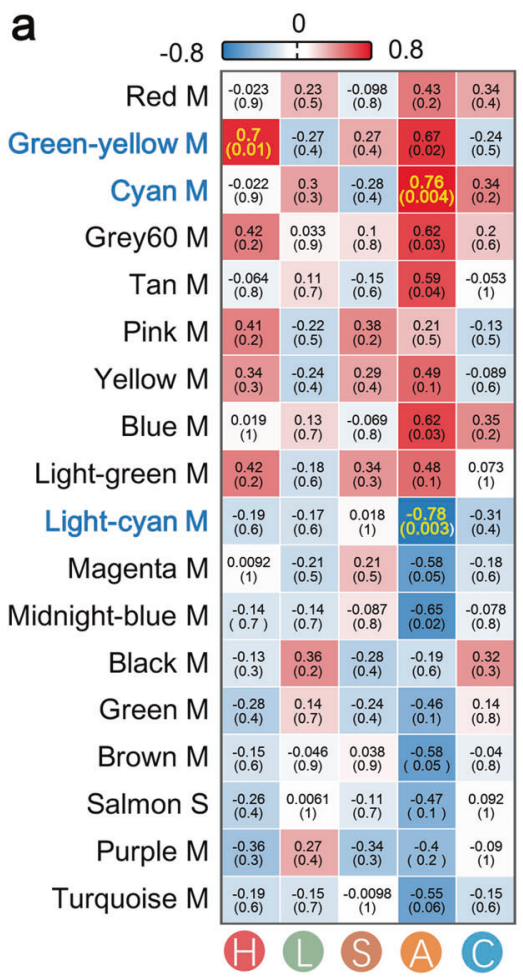

Fig. 4 Metagenomic modules correlate with depressive-like (DL) phenotypes in $M$. fascicularis. a Association of metagenomic WGCNA modules with DL behaviors. Heatmap of the correlation coefficients of 18 modules (each module (M) is listed on the left side of the image) and behavioral phenotype indices ( $\mathrm{H}$ huddle, L locomotion, $\mathrm{S}$ sit alone, A amicable, $\mathrm{C}$ communication) located at the bottom of the image. Red and blue squares indicate positive and negative associations, respectively. The Spearman's correlation coefficient and $P$ value are shown within the squares (yellow bold

total, 559 differentially expressed metabolites (VIP $>1.0$, $P<0.05$ ) between the two groups were identified (Supplementary Table $4 \mathrm{a}-\mathrm{g}$ ). As the samples of MGB axis were diverse, these detected samples shared only a limited number of metabolites (Supplementary Fig. 4a, b). As such, it is likely that different components of a given metabolic pathway synergistically regulate the function of MGB axis in different tissues. Thus, WGCNA was used to identify the metabolic modules of the MGB axis. This analysis clustered the 559 metabolites into eight different modules (Fig. 5a and Supplementary Table 5a), in which four modules (blue M, black M, yellow $M$, and green M; Fig. 5b-d) were significantly correlated with at least one index of DL behaviors $(P<0.01$, Fig. 5a and Supplementary Table 5b). The blue M $(r=-0.91, P<0.001$; Fig. $5 c)$, the largest one, was associated with four behaviors: huddle, locomotion, sit alone, and communication; the metabolites that comprised the blue $\mathrm{M}$ were mainly involved in peripheral and central fatty acyl, glycerophospholipid and sphingolipid metabolism within the MGB axis. For the other three modules, the primary metabolites were also consistently related to these three metabolic b

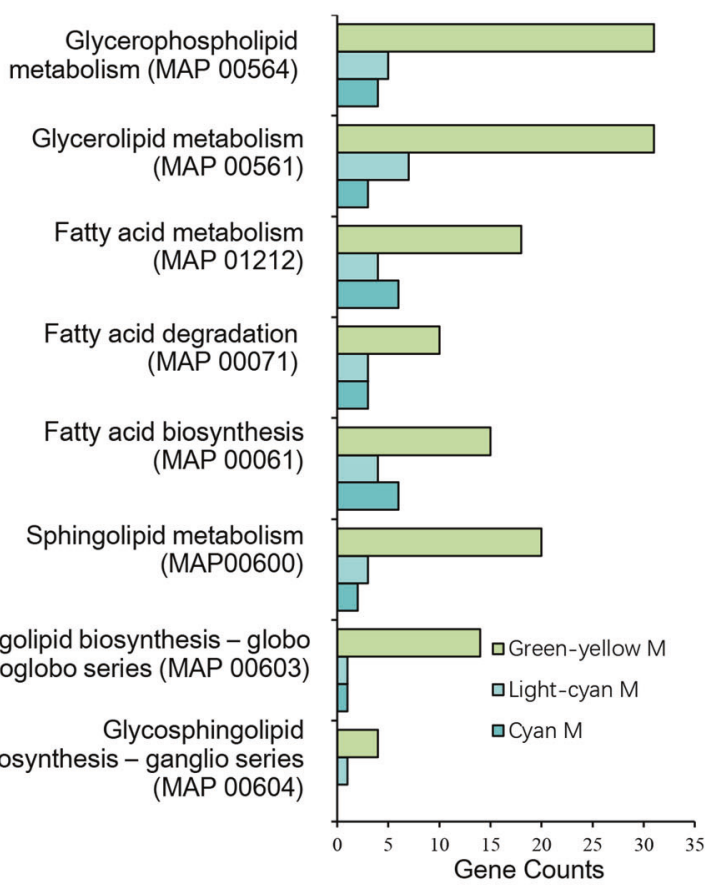

font indicates $P \leq 0.01)$. Three metagenomic modules including Green-yellow M, Cyan M, and Light-cyan M were significantly linked with depressive behaviors. b Lipid-related pathways enriched from three metagenomic WGCNA modules (Green-yellow M, Cyan M, and Light-cyan M). Pathways were annotated using the Kyoto Encyclopedia of Genes and Genomes (KEGG), and were enriched for fatty acyl, sphingolipid metabolism, and glycerophospholipid metabolism. The bar length represents the gene counts annotated into the same metabolic pathway in each module.

pathways. These findings were similar to what we found in the metagenomic WGCNA analysis, suggesting that disturbances of the gut microbiome may contribute to the onset of DL behaviors through modulating peripheral and central fatty acyl, sphingolipid and glycerophospholipid metabolism in monkeys.

\section{Alterations of glycerophospholipid metabolism with a region-specific manner in DL monkeys}

Using WGCNA analysis, we described above relationships between gut microbiome and disturbed peripheral and central lipids metabolism in DL macaques. To further outline which canonical metabolic pathways were mainly modulated by gut microbiome at the functional levels, all detected differential metabolites of lipid metabolism in the three brain regions (hippocampus, prefrontal cortex, and amygdala) were mapped to the KEGG database in a manually curated manner. Consequently, we found that the hippocampus was the area with the highest degree of disrupted lipid metabolic pathways (Fig. 6a). Interestingly, 
a

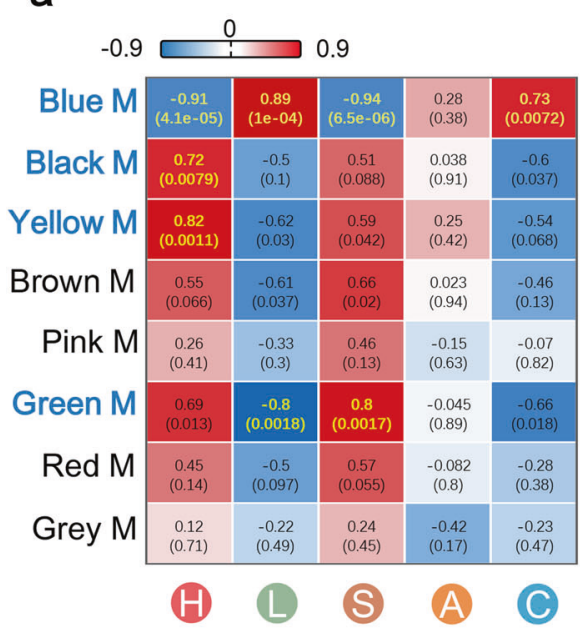

C

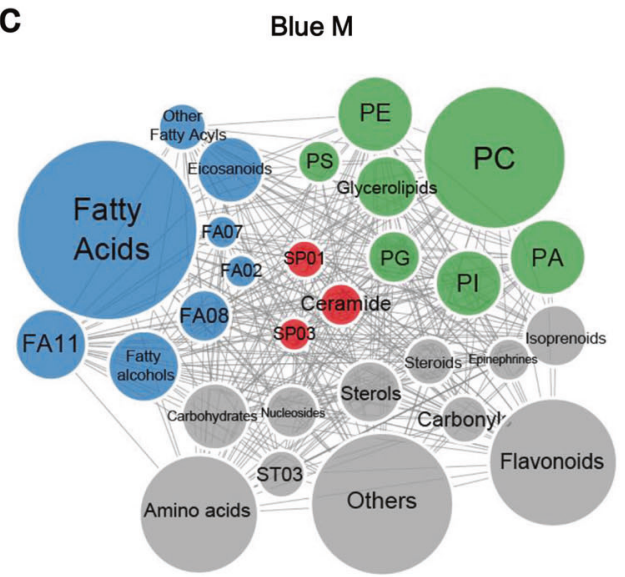

Fig. 5 Metabolomic correlations with behavioral phenotypes in depressive-like (DL) M. fascicularis. a Associations of metabolomic modules with behavioral phenotype indexes. Heatmap of the correlation coefficients and eight modules [each module (M) is listed at the left side of the image] and behavioral phenotype indices ( $\mathrm{H}$ huddle, $\mathrm{L}$ locomotion, $\mathrm{S}$ sit alone, A amicable, $\mathrm{C}$ communication shown at the top of the image). Red and blue squares indicate positive and negative associations, respectively. The Spearman's correlation coefficient and $P$ value are shown within the squares (yellow bold font indicates $P<$

these hippocampal differential metabolites were mainly enriched in glycerophospholipid metabolism, accompanying with few metabolites belonging to sphingolipid metabolism (Fig. 6b). The majority of hippocampal metabolites in glycerophospholipid metabolism were upregulated in DL group compared with HC group. Meanwhile, two metabolites (dihydroceramide and ceramide-1-phosphate) involved in sphingolipid metabolism were significantly decreased in the DLs relative to HCs. These findings suggested that imbalances of hippocampal sphingolipid and glycerophospholipid metabolism were linked with DL monkeys. Together, our finding revealed that the dysbiosis of gut microbiome may participate in the onset of depressive
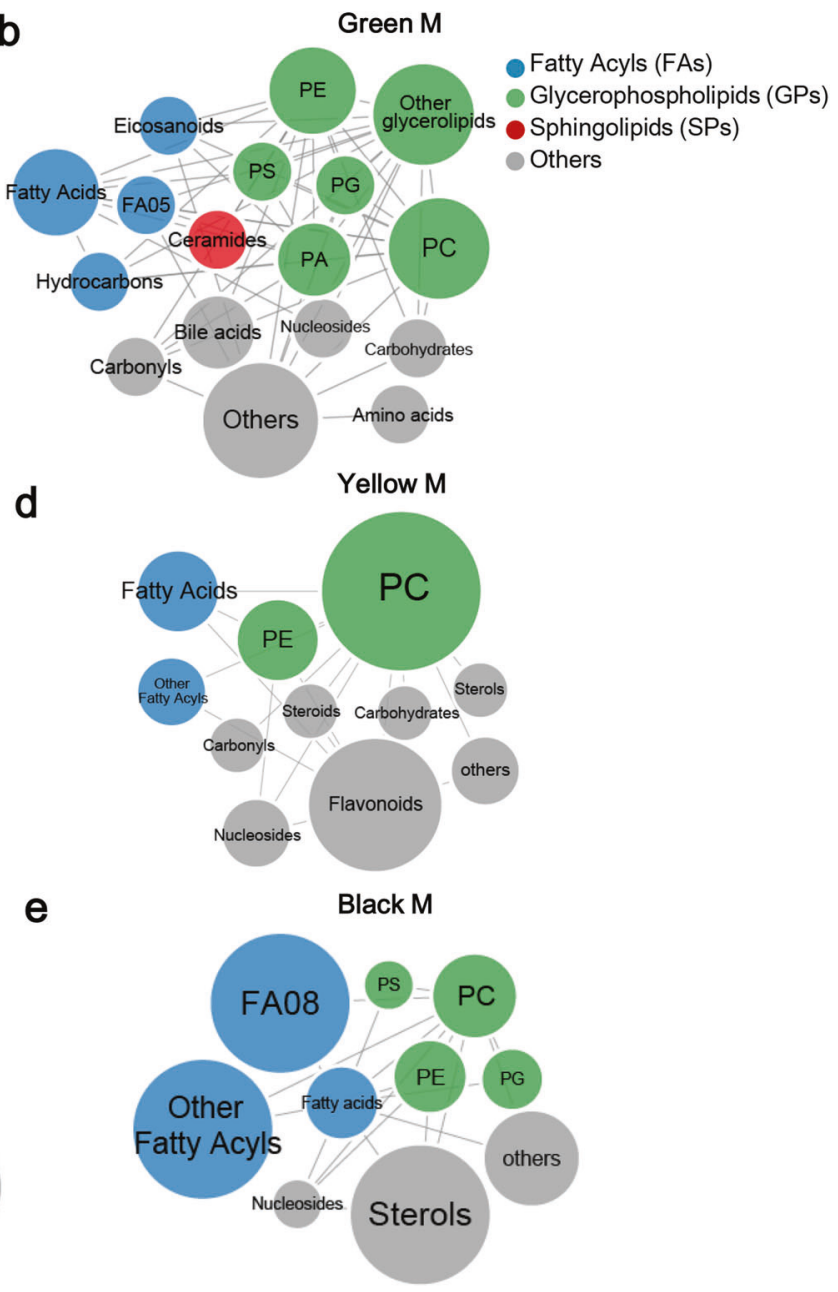

0.01). b-e Network diagrams of differential metabolites in four metabolic modules (blue $\mathrm{M}$, yellow $\mathrm{M}$, green $\mathrm{M}$, and black $\mathrm{M}$ ) that are significantly correlated to DL behaviors $(P<0.01)$. Circle colors indicate the different kinds of lipids in each module; circle size indicates the abundance of the metabolites. Metabolites were mainly classified into three classes: fatty acyls (FA), sphingolipids (SPs), and Glycerophospholipids (GPs). PA phosphatidic acid, PE phosphatidylethanolamine, PS phosphoserine, PC phosphatidylcholine, PI phosphatidylinositol, and PG phosphatidylglycerol.

behaviors by modulating the gut-brain glycerophospholipid metabolism in a region-specific manner.

\section{Discussion}

Growing evidence suggests that disturbed gut microbiome may contribute to depression pathology, but the specific mechanisms remain unclear. Here we observed that DL macaque is characterized by alterations of microbial composition, function, and metabolic pathways of the MGB axis. The altered microbial and metabolic modules linked the gut microbiome with dysregulation of 
a

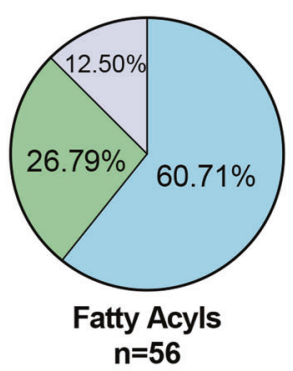

b

\section{Fatty Acyls}

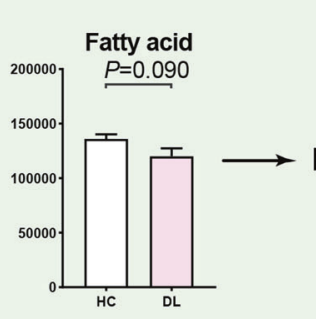

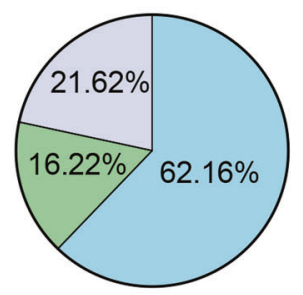

Glycerophospholipids $n=37$

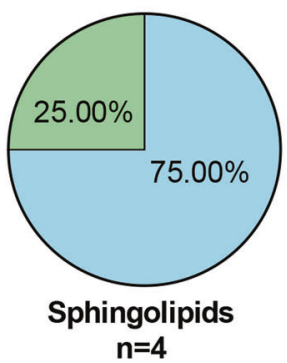

Hippocampus

PFC

AMY

\section{Sphingolipid metabolism}

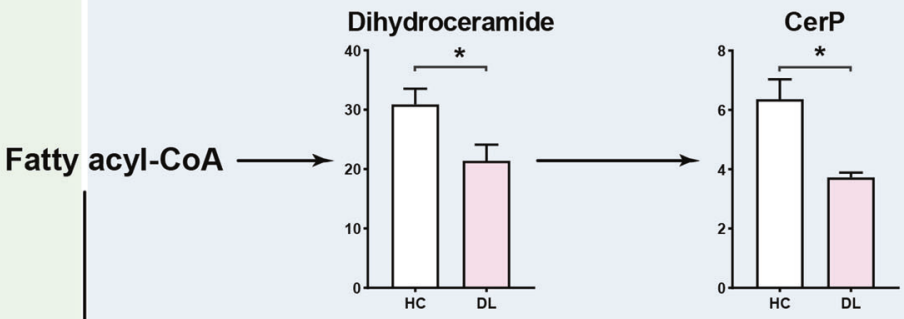

\section{Glycerophospholipid metabolism}
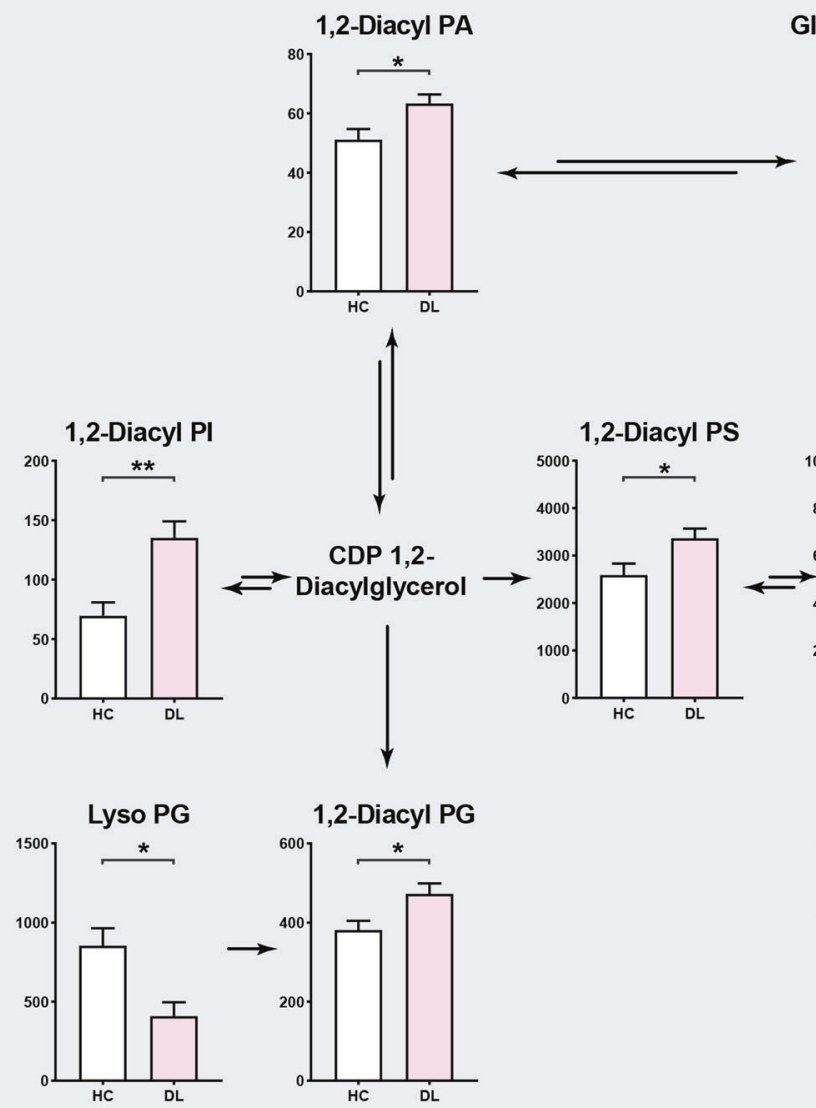

Glycerophosphocholine

Lyso PC

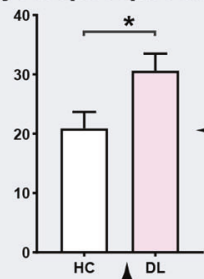

HC $\uparrow$
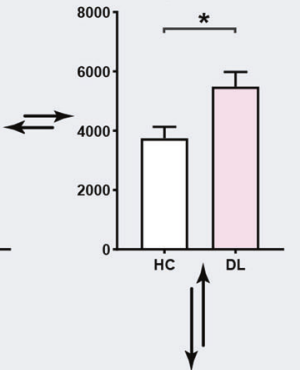

2-Diacyl PS

1,2-Diacyl PE

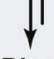

$\downarrow$

1,2-Diacyl PC
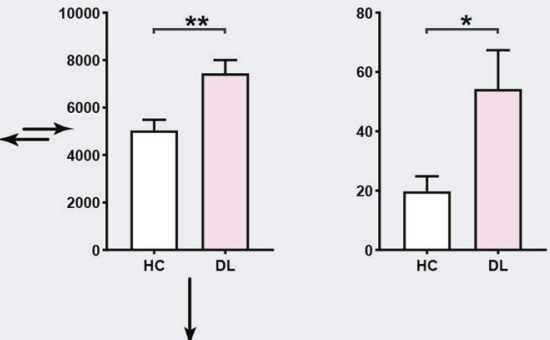

Lyso PE

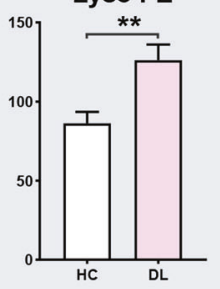

peripheral and central fatty acyl, sphingolipid, and glycerophospholipid metabolism in the DL monkeys. Among the detected brain tissues, disturbances of hippocampal glycerophospholipid metabolism were a hallmark of DL monkeys. To our knowledge, this is the first use of a nonhuman primate model to reveal that gut microbiome 
4 Fig. 6 Disturbance of hippocampal glycerophospholipid metabolism in depressive-like (DL) M. fascicularis. a Disturbances of fatty acyls (FA), sphingolipids (SPs), and glycerophospholipids (GPs) showing a region-specific manner in DL $M$. fascicularis. The three kinds of lipids were substantially changed in the hippocampus, accounting for ranging from $60.71-75 \%$ of detected differential metabolites in the three brain regions: hippocampus, prefrontal cortex (PFC), and amygdala (AMY). b These hippocampal differential metabolites were mainly mapped into the glycerophospholipid metabolism. Majority of metabolites in the glycerophospholipid metabolism were upregulated in the DL group relative to the HC group. Meanwhile, two metabolites related to sphingolipid metabolism were depleted in the DL group. These findings suggest that disturbance of hippocampal glycerophospholipid metabolism was a hallmark of DL, which may involve in imbalance of hippocampal sphingolipid and glycerophospholipid metabolism. CerP ceramide-1-phosphate, PA phosphatidic acid, PC phosphatidylcholine, PI phosphatidylinositol, PS phosphatidylserine, PE phosphatidylethanolamine, PG phosphatidylglycerol.

disturbances may mediate DL behaviors by affecting the host's MGB metabolism.

Previously, several clinical investigations have observed significant gut microbiome changes in patients with MDD. These clinical findings were diverse and partly inconsistent due to differences in recruitment standards, sequencing, and analytical methods. Overall, at the phylum level, disturbances of Firmicutes and Bacteroidetes were the hallmark of depression in those investigations $[19,35,36]$. In addition, up- and downregulated family Enterobacteriaceae were linked with depression with different severity $[19,36]$. At the genus level, the abundances of Coprococcus and Dialister were depleted in general practitioner-reported depression [37], but not in drug-free patients with MDD [20]. Interestingly, we found that genus Coprococcus and Dialister were two of microbial markers that distinguish patients with MDD and bipolar disorder [20]. These findings suggest that further metagenomic studies should be performed to identify the specific microbial species belonging to the two genera in depression. Consistent with clinical findings, we found that microbial composition of DL monkeys were substantially different from that of control monkeys, which may result from the continual stress experienced in the DL monkeys. In addition, using a rodent model of social defeat, Pearson-Leary et al. [38], also revealed that stress resulted in significant changes of microbial composition in DL rats relative to their pre-stress status. These data suggest that stress may attribute to the onset of depressive behaviors by shaping the gut microbiome. Here, we found that the DL monkeys were characterized by increased Veillonellaceae and Lachnospiraceae OTUs and decreased Ruminococcaceae OTUs. Ruminococcaceae have been linked with the maintenance of gut health; therefore, decreased Ruminococcaceae suggests abnormal microbial status in the DL monkey. Interestingly, the majority of OTUs (89.28\%) belong to phylum Firmicutes. In addition, we observed that
50 differential MGS between the DL and HC groups were primarily concentrated in Firmicutes (89.6\%). In line with these findings, we previously reported that these OTUs that reliably differentiated MDD from HCs also belonged primarily to Firmicutes (76.7\%) [19]. Thus, both human and nonhuman primate results have consistently demonstrated that disturbances of the bacterial phylum Firmicutes may be a hallmark of depression.

The brain is particularly enriched in lipids with a diverse lipid composition compared to other tissues [39]. Lipids have an important role in neuronal function, and lipid composition of the brain may influence perception and emotional behavior, which may lead to depression and anxiety disorders [40-42]. Previous preclinical and clinical experiments have found that depression is characterized by disturbances of peripheral and central lipid metabolism [43-46], but its role in the pathogenesis of depression is not explicitly clear. Here we found that both altered microbial and metabolic modules involving fatty acyl, sphingolipid, and glycerophospholipid metabolism were highly correlated with DL behaviors. In these microbial modules, we identified several microbial genes which were involved in the fatty acyl, sphingolipid, and glycerophospholipid metabolism, suggesting that gut microbes and their modulated host's metabolites may play critical roles in depression pathology. In line with our current findings, Zhang et al. [47] found differential lipid metabolism disturbance in the hippocampus in the chronic unpredictable mild stress rat model of depression. They also found decreased expression of lipid metabolism-related enzyme related to fatty acid synthesis and metabolism, and in glycerophospholipid metabolism. Oliveira et al. [48] also found that chronic stress resulted in lipidomic changes with a region-specific manner in the depressive rats. However, disturbances of lipid metabolism in the prefrontal cortex were more obvious than that in the hippocampus of the DL rats. In addition, they found that phospholipid metabolism, but not glycerophospholipids metabolism, were mainly disturbed in their depression model. These mild differences may result from different depression models used in rodents and primates. Together, these studies have shown that lipid metabolism in the prefrontal cortex and hippocampus was associated with depression.

In addition, we found that altered hippocampal glycerophospholipids metabolism was a prominent feature of DL monkeys. Here, the majority of hippocampal metabolites involved in glycerophospholipids metabolism were enriched, but two metabolites (dihydroceramide and ceramide1-phosphate) involving the phospholipid metabolism were depleted in depressive monkeys relative to controls. These findings suggest that disturbance of hippocampal glycerophospholipid metabolism may involve an imbalance of hippocampal sphingolipid and glycerophospholipid 
metabolism. The CNS contains three major classes of lipids: phospholipid, sphingolipid, and cholesterol [39]. Sphingolipids participate in the development and differentiation of neuronal membranes. Phospholipids, particularly glycerophospholipids, are critical components of neuronal membranes and myelin, and principal regulators of synaptic function $[49,50]$. Hence these findings may account for white matter abnormalities and synaptic dysfunction that frequently accompany and likely contribute to MDD $[51,52]$. Interestingly, the majority of polyunsaturated fatty acids used for brain neural membrane glycerophospholipid synthesis are derived from the gastrointestinal tract not CNS. Consistent with these findings, we showed that MGB axis' glycerophospholipid metabolism was significantly disturbed in depressive monkey.

It should be acknowledged that there are some limitations of this study: (i) the functional significance of the candidate gut microbes related to depression requires further investigation, to identify key microbial species that may contribute to the onset of depression; (ii) in addition to metabolic pathways, how the gut microbiome attributes to DL behaviors in monkeys through the vagus nerve, hypothalamic-pituitary-adrenal axis and immune system remains largely unidentified, which is worthy of further investigation as these pathways may be implicated in the MGB crosstalk in depression; (iii) here only female monkeys were studied, thus sex-bias cannot be ruled out. However, this model requires the use of female M. fascicularis only because the majority of animals-and those that form the natural social hierarchy and develop DL symptoms-within the social unit are females; (iv) we outlined that disturbances of MGB axis' glycerophospholipid metabolism were implicated in depression. Future studies to specifically interrogate these pathways are required to further clarify its function and discover new therapeutic targets; (v) the sample size of this study is relatively small. Thus, the reliability of the correlations reported may be impacted. Further longitudinal studies with larger samples should be performed to determinate how the gut microbes and their modulated metabolic pathways dynamically change in the depressive monkeys.

Taken together, using a newly developed nonhuman primate model, we found that $M$. fascicularis displaying naturally occurring DL behaviors were characterized by alterations of microbial composition, function, and MGB axis metabolism, which advances our understanding of how the gut microbiome may contribute to depression pathology.

Author contributions Designed the experiments: PX and JL. Performed the metagenomic analysis: PZ, JW, HPZ, and YFL. Performed the metabolomic analysis: PZ, JW, YFL, and JJD. Analyzed the metagenomic and metabolomic data: PZ, JW, XMT, TJC, and H.P.Z. Animal behaviors: BMY, WWL, and YH. Drafted the paper: PX and PZ. Revised the paper for intellectual content: PX, JL, SWP, and MLW.
Funding This work was supported by the National Key R\&D Program of China (2017YFA0505700, 2016YFC1307200), Non-profit Central Research Institute Fund of Chinese Academy of Medical Sciences (2019PT320002), Projects of International Cooperation and Exchanges NSFC (81820108015), the Natural Science Foundation Project of China (81971296, 81771490, 81371310, and 81200899), Chongqing Science \& Technology Commission (cstc 2019 jcyjjqX0009), and institutional funds from the State University of New York (SUNY) Upstate Medical University. This paper is subject to the SUNY Open Access Policy.

\section{Compliance with ethical standards}

Conflict of interest The authors declare that they have no conflict of interest.

Publisher's note Springer Nature remains neutral with regard to jurisdictional claims in published maps and institutional affiliations.

\section{References}

1. Frankish H, Boyce N, Horton R. Mental health for all: a global goal. Lancet. 2018;392:1493-4.

2. Yano JM, Yu K, Donaldson GP, Shastri GG, Ann P, Ma L, et al. Indigenous bacteria from the gut microbiota regulate host serotonin biosynthesis. Cell. 2015;161:264-76.

3. Warden D, Rush AJ, Trivedi MH, Fava M, Wisniewski SR. The STAR*D Project results: a comprehensive review of findings. Curr psychiatry Rep. 2007;9:449-59.

4. Ruelaz AR. Treatment-resistant depression: strategies for management. 2006;23:34-7.

5. Willner P. Validity, reliability and utility of the chronic mild stress model of depression: a 10-year review and evaluation. Psychopharmacology. 1997;134:319-29.

6. Willner P. Chronic mild stress (CMS) revisited: consistency and behavioural-neurobiological concordance in the effects of CMS Neuropsychobiology. 2005;52:90-110.

7. Canuto A, Weber K, Baertschi M, Andreas S, Volkert J, Dehoust $\mathrm{MC}$, et al. Anxiety disorders in old age: psychiatric comorbidities, quality of life, and prevalence according to age, gender, and country. Am J Geriatr Psychiatry. 2018;26:174-85.

8. Hassard J, Teoh KRH, Visockaite G, Dewe P, Cox T. The cost of work-related stress to society: a systematic review. J Occup Health Psychol. 2018;23:1-17.

9. Sheikh MA. The potential protective effect of friendship on the association between childhood adversity and psychological distress in adulthood: a retrospective, preliminary, three-wave population-based study. J Affect Disord. 2018;226:21-7.

10. Xu F, Wu Q, Xie L, Gong W, Zhang J, Zheng P, et al. Macaques exhibit a naturally-occurring depression similar to humans. Sci Rep. 2015;5:9220.

11. Li X, Xu F, Xie L, Ji Y, Cheng K, Zhou Q, et al. Depression-like behavioral phenotypes by social and social plus visual isolation in the adult female Macaca fascicularis. PloS ONE. 2013;8: e73293.

12. Shively CA, Laber-Laird K, Anton RF. Behavior and physiology of social stress and depression in female cynomolgus monkeys. Biol Psychiatry. 1997;41:871.

13. Lea AJ, Akinyi MY, Nyakundi R, Mareri P, Nyundo F, Kariuki T, et al. Dominance rank-associated gene expression is widespread, sex-specific, and a precursor to high social status in wild male baboons. Proc Natl Acad Sci. 2018;115:E12163-71.

14. Snyder-Mackler N, Sanz J, Kohn JN, Voyles T, Pique-Regi R, Wilson ME, et al. Social status alters chromatin accessibility and 
the gene regulatory response to glucocorticoid stimulation in rhesus macaques. Proc Natl Acad Sci. 2019;116:1219-28.

15. Keeney A, Jessop DS, Harbuz MS, Marsden CA, Hogg S, Blackburn-Munro RE. Differential effects of acute and chronic social defeat stress on hypothalamic-pituitary-adrenal axis function and hippocampal serotonin release in mice. J Neuroendocrinol. 2006;18:330-8.

16. Wood SK, Wood CS, Lombard CM, Lee CS, Zhang XY, Finnell $\mathrm{JE}$, et al. Inflammatory factors mediate vulnerability to a social stress-induced depressive-like phenotype in passive coping rats. Biol Psychiatry. 2015;78:38-48.

17. Kelly JR, Borre Y, Brien CO, Patterson E, Aidy SE, Deane J, et al. Transferring the blues: depression-associated gut microbiota induces neurobehavioural changes in the rat. J Psychiatr Res. 2016;82:109-18.

18. Cryan JF, O'Riordan KJ, Cowan CSM, Sandhu KV, Bastiaanssen TFS, Boehme M, et al. The Microbiota-Gut-Brain Axis. Physiol Rev. 2019;99:1877-2013.

19. Zheng P, Zeng B, Zhou C, Liu M, Fang Z, Xu X, et al. Gut microbiome remodeling induces depressive-like behaviors through a pathway mediated by the host's metabolism. Mol Psychiatry. 2016; 21:786-96.

20. Zheng P, Yang J, Li Y, Wu J, Liang W, Yin B, et al. Gut microbial signatures can discriminate unipolar from bipolar depression. Adv Sci. 2020:1902862.

21. Zheng P, Zeng B, Liu M, Chen J, Pan J, Han Y, et al. The gut microbiome from patients with schizophrenia modulates the glutamate-glutamine-GABA cycle and schizophrenia-relevant behaviors in mice. Sci Adv. 2019;5:eaau8317.

22. Li X, Liang S, Xia Z, Qu J, Liu H, Liu C et al. Establishment of a Macaca fascicularis gut microbiome gene catalog and comparison with the human, pig, and mouse gut microbiomes. GigaScience. 2018;7:giy100.

23. Menzel P, Ng KL, Krogh A. Fast and sensitive taxonomic classification for metagenomics with Kaiju. Nat Commun. 2016;7:11257.

24. Bradley W, Steven H, Robert P. Utilities for quantifying separation in PCA/PLS-DA scores plots. Anal Biochem. 2013;433:102-4.

25. Langfelder P, Horvath S. WGCNA: an R package for weighted correlation network analysis. BMC Bioinforma. 2008;9:559.

26. Sousa AMM, Zhu Y, Raghanti MA, Kitchen RR, Onorati M, Tebbenkamp ATN, et al. Molecular and cellular reorganization of neural circuits in the human lineage. Science. 2017;358:1027-32.

27. Weatheall $\mathrm{D}$. The use of non-human primates in research. London: Academy of Medical Sciences; 2006.

28. Kikuchi T, Morizane A, Doi D, Magotani H, Onoe H, Hayashi T, et al. Human iPS cell-derived dopaminergic neurons function in a primate Parkinson's disease model. Nature. 2017;548:592-6.

29. Chu X. Preliminary validation of natural depression in macaques with acute treatments of the fast-acting antidepressant ketamine. Behavioural Brain Res. 2019;360:60-8.

30. Zheng P, Li Y, Wu J, Zhang H, Huang Y, Tan X, et al. Perturbed microbial ecology in myasthenia gravis: evidence from the gut microbiome and fecal metabolome. Adv Sci. 2019;6:1901441.

31. Bolger AM, Lohse M, Usadel B. Trimmomatic: a flexible trimmer for Illumina sequence data. Bioinformatics. 2014;30:2114-20.

32. Newell C, Bomhof MR, Reimer RA, Hittel DS, Rho JM, Shearer J. Ketogenic diet modifies the gut microbiota in a murine model of autism spectrum disorder. Mol Autism. 2016;7:37.

33. Segata N, Izard J, Waldron L, Gevers D, Miropolsky L, Garrett WS, et al. Metagenomic biomarker discovery and explanation. Genome Biol. 2011;12:R60.
34. Nielsen HB, Almeida M, Juncker AS, Rasmussen S, Li J, Sunagawa $\mathrm{S}$, et al. Identification and assembly of genomes and genetic elements in complex metagenomic samples without using reference genomes. Nat Biotechnol. 2014;32:822-8.

35. Naseribafrouei A, Hestad K, Avershina E, Sekelja M, Linlokken A, Wilson R, et al. Correlation between the human fecal microbiota and depression. Neurogastroenterol Motil: Off J Eur Gastrointest Motil Soc. 2014;26:1155-62.

36. Jiang H, Ling Z, Zhang Y, Mao H, Ma Z, Yin Y, et al. Altered fecal microbiota composition in patients with major depressive disorder. Brain Behav Immun. 2015;48:186-94.

37. Valles-Colomer M, Falony G, Darzi Y, Tigchelaar EF, Wang J, Tito $\mathrm{RY}$, et al. The neuroactive potential of the human gut microbiota in quality of life and depression. Nat Microbiol. 2019;4:623-32.

38. Pearson-Leary J, Zhao C, Bittinger K, Eacret D, Luz S, Vigderman AS, et al. The gut microbiome regulates the increases in depressive-type behaviors and in inflammatory processes in the ventral hippocampus of stress vulnerable rats. Molecular psychiatry. 2019;4:1-2.

39. Bozek K, Wei Y, Yan Z, Liu X, Xiong J, Sugimoto M, et al. Organization and evolution of brain lipidome revealed by largescale analysis of human, chimpanzee, macaque, and mouse tissues. Neuron. 2015;85:695-702.

40. Yadav RS, Tiwari NK. Lipid integration in neurodegeneration: an overview of Alzheimer's disease. Mol Neurobiol. 2014;50:168-76.

41. Kornhuber J, Rhein C, Müller CP, Mühle C. Secretory sphingomyelinase in health and disease. Biol Chem. 2015;396:707-36.

42. Adibhatla RM, Hatcher JF. Phospholipase A(2), reactive oxygen species, and lipid peroxidation in CNS pathologies. BMB Rep. 2008;41:560-7.

43. Liu X, Li J, Zheng P, Zhao X, Zhou C, Hu C, et al. Plasma lipidomics reveals potential lipid markers of major depressive disorder. Anal Bioanal Chem. 2016;408:6497-507.

44. Liu X, Zheng P, Zhao X, Zhang Y, Hu C, Li J, et al. Discovery and validation of plasma biomarkers for major depressive disorder classification based on liquid chromatography-mass spectrometry. J Proteome Res. 2015;14:2322-30.

45. Zheng P, Gao HC, Li Q, Shao WH, Zhang ML, Cheng K, et al. Plasma metabonomics as a novel diagnostic approach for major depressive disorder. J Proteome Res. 2012;11:1741-8.

46. Jia HM, Li Q, Zhou C, Yu M, Yang Y, Zhang HW, et al. Chronic unpredictive mild stress leads to altered hepatic metabolic profile and gene expression. Sci Rep. 2016;6:23441.

47. Zhang Y, Yuan S, Pu J, Yang L, Zhou X, Liu L, et al. Integrated metabolomics and proteomics analysis of hippocampus in a rat model of depression. Neuroscience. 2018;371:207-20.

48. Oliveira TG, Chan RB, Bravo FV, Miranda A, Silva RR, Zhou B, et al. The impact of chronic stress on the rat brain lipidome. Mol Psychiatry. 2016;21:80-88.

49. Romme IA, de Reus MA, Ophoff RA, Kahn RS, van den Heuvel MP. Connectome disconnectivity and cortical gene expression in patients with schizophrenia. Biol Psychiatry. 2017;81:495-502.

50. Lee JC, Park SM, Kim IY, Sung H, Seong JK, Moon MH. Highfat diet-induced lipidome perturbations in the cortex, hippocampus, hypothalamus, and olfactory bulb of mice. Biochim biophys Acta Mol cell Biol lipids. 2018;1863:980-90.

51. Sacchet MD, Gotlib IH. Myelination of the brain in major depressive disorder: an in vivo quantitative magnetic resonance imaging study. Sci Rep. 2017;7:2200.

52. Fields RD. White matter in learning, cognition and psychiatric disorders. Trends Neurosci. 2008;31:361-70. 